\title{
La strada verso un servizio sanitario più sicuro non passa attraverso la blame culture
}

\author{
Riccardo Tartaglia ${ }^{1}$
}

${ }^{1}$ Centro Gestione Rischio Clinico e Sicurezza del Paziente, Regione Toscana

La strada verso un servizio sanitario più sicuro è un percorso lungo e difficile che però ormai molti operatori sanitari stanno percorrendo con grande passione e coinvolgimento. Il problema della sicurezza del paziente, e della malpractice, lo sentono tutti sulla propria pelle.

Per molti professionisti il tema del rischio clinico ha rappresentato la via pragmatica alla qualità. Rispetto ad un processo di accreditamento che non era stato pienamente compreso da tutti nel suo significato, la gestione del rischio è riuscita da subito a catalizzare l'interesse degli operatori e dei pazienti su problemi concreti e facilmente percepibili.

È necessario però essere consapevoli, se l'obiettivo che ci si prefigge è quello della sicurezza del paziente, che la cultura della colpa (blame culture) non serve a rendere la pratica clinica più sicura.

Più gli operatori sanitari temono le conseguenze dei loro errori, più tendono a nasconderli o ad attribuirne la causa ad altri. Più si colpevolizzano gli operatori di prima linea, più le organizzazioni sanitarie diventano vulnerabili agli incidenti. Questo è quanto affermano importanti istituzioni sanitarie ed esperti di errori medici a livello internazionale. Oggi i servizi sanitari più evoluti e impegnati per garantire la sicurezza del paziente prevedono la possibilità di segnalare e imparare dagli errori: ciò non significa ovviamente garantire l'impunità di chi ha sbagliato, ma favorire le occasioni di discussione tra operatori sanitari (gli audit clinici e le rassegne di morbilità e mortalità) senza il rischio che la segnalazione dell'errore, interna al sistema, sia utilizzata per denunciare chi l'ha fatta. La legge italiana non consente ad oggi lo sviluppo di una tale cultura, essendoci l'elevata probabilità per il Sanitario che segnala un proprio errore di finire davanti al magistrato dato che è previsto l'obbligo, per chi viene a conoscenza di un errore medico, di denunciarlo.

La paura delle conseguenze giudiziarie impedisce inoltre anche la segnalazione dei mancati inciden- ti, molto più numerosi degli eventi avversi che assurgono all'onore delle cronache. I mancati incidenti potrebbero al contrario rappresentare delle importanti lezioni per migliorare la sicurezza e introdurre nel sistema delle barriere protettive per impedire all'errore di ripetersi.

Per sviluppare una cultura del "segnalare e imparare dagli errori” è necessario partire dal dato di fatto che l'errore non può essere evitato, poiché è insito nelle limitate capacità sensoriali, attentive $\mathrm{e}$ razionali dell'essere umano, ma è invece possibile impedire che giunga a cagionare un danno.

Bisogna, al di là delle tante certezze reclamizzate dai mass media, rendersi conto che la medicina non è una scienza esatta ma una disciplina biologica e, come tale, basata su un livello di variabilità tale da permettere solo valutazioni di tipo probabilistico, sia per quanto riguarda la diagnosi che la terapia. È importante sottolineare che la variabilità biologica non riguarda solo il paziente, ma anche l'operatore sanitario, per il quale l'attività professionale non è solo l'applicazione di procedure predefinite ma un processo mentale assolutamente personale legato alla propria esperienza, alla capacità elaborativa e allo specifico contesto organizzativo e sociale.

Se il cambiamento culturale che porta a un'evoluzione dalla blame culture alla reporting and learning culture è una condizione indispensabile nel percorso per realizzare un servizio sanitario più sicuro, sono anche altri gli obiettivi da raggiungere.

Innanzitutto è importante dare un assetto organizzativo alla gestione del rischio clinico. Questo non significa costituire nuove strutture (quelle esistenti che si occupano di qualità riescono ad assolvere nella maggioranza dei casi anche a questo compito), bensì individuare all'interno delle aziende sanitarie un ruolo, una figura professionale che si occupi della sicurezza del paziente e rappresenti un punto di riferimento non solo per il cittadino, ma anche per il sistema assicurativo. 
A tale scopo è necessario formare del personale con competenze specifiche che non sono ancora presenti nelle nostre aziende, favorendo ad esempio le conoscenze nel campo del fattore umano, della psicologia delle organizzazioni e delle scienze della comunicazione.

Altrettanto importante è poter disporre di banche dati sui sinistri. Sino ad oggi questo tipo di informazione era patrimonio esclusivo delle compagnie assicurative. Le aziende sanitarie devono invece poter avere dei propri dati sulla sinistrosità che consentano un confronto e un contraddittorio tra pari con gli assicuratori.

Un'altra tappa essenziale del percorso è la creazione di sistemi di incident reporting. Quelli attualmente in uso si basano spesso su modelli di riferimento che differiscono troppo dalle realtà operative, ed è quindi necessario svilupparne di coerenti alle caratteristiche sociali e storiche degli specifici contesti assistenziali. L'obiettivo dei sistemi di incident reporting deve essere comunque quello di diffondere la pratica dell'audit clinico e delle rassegne di mortalità e morbilità, utili occasioni di approfondimento degli eventi avversi e delle disfunzioni organizzative.

La promozione delle buone pratiche di lavoro, basata sulle evidenze della letteratura, è un ulteriore passo verso il raggiungimento di un servizio sanitario più sicuro. Di recente la Regione Toscana, anche in considerazione dei tempi lunghi necessari per l'effettiva creazione di una cultura del "segnalare e dell'imparare dagli errori”, ha istituito un sistema di campagne per la sicurezza del paziente incentrate su temi specifici: tali campagne cercano di dare una risposta immediata ad alcune delle problematiche più segnalate. Inoltre consentono di valorizzare e rendere visibili i risultati ottenuti dalle strutture che si impegnano per la sicurezza dei pazienti, oltre che di contenere i costi assicurativi mediante una verifica dei livelli raggiunti nella gestione del rischio clinico. Le campagne stanno favorendo la messa punto di buone pratiche per la sicurezza del paziente che è nostro interesse rendere visibili al cittadino, anche mediante un sistema di certificazione volontaria.

Anche l'ergonomia (fattore umano) è un ambito nel quale è importante intervenire: ad esempio, nell'ottica della prevenzione degli errori umani all'interno dei processi di lavoro, è imprescindibile il controllo delle apparecchiature biomedicali, mentre ancora oggi sono numerosi gli strumenti di uso comune che non hanno ricevuto adeguati test di usabilità. All'interno di questa problematica si inserisce anche la corretta organizzazione e formazione dei gruppi di lavoro: andrebbe favorita una formazione interdisciplinare che abitui i team a lavorare insieme.

Lungo questo percorso bisogna essere consapevoli che gli incidenti saranno comunque presenti e prendere atto che la sicurezza non è mai raggiunta una volta per tutte.

La nostra Regione, che pure è stata una delle prime ad occuparsi di gestione del rischio clinico (istituendo un apposito centro) e che presenta un indice di sinistrosità al di sotto della media nazionale, è stata recentemente colpita da un grave evento avverso: il trapianto di organi provenienti da un donatore sieropositivo al HIV a tre riceventi.

Alla luce di quanto avvenuto, riteniamo sia doveroso intervenire anche in quelle aree in cui il lavoro è estremamente proceduralizzato e svolto con tecnologie avanzate.

In queste situazioni è necessario effettuare attente verifiche e, nella stesura delle procedure di lavoro, prendere in adeguata considerazione il fattore umano, poiché anche in questi casi ci possono essere delle criticità più o meno manifeste.

Gli obiettivi che ci prefiggiamo per il prossimo futuro possono essere sintetizzati nei seguenti punti:

- ridurre i livelli di sinistrosità attraverso l'apprendimento di una cultura del "segnalare e imparare dagli errori” e l'applicazione delle buone pratiche di lavoro;

- coinvolgere il cittadino nei programmi di miglioramento della sicurezza;

- migliorare la comunicazione con il paziente e tra operatori;

- attivare la logica dell'attore equivalente;

- lavorare in un "clima organizzativo" in cui oltre ai pazienti siano soddisfatti gli operatori.

Sulla base di quanto sta emergendo dalla ricerca in corso ad opera dell'Agenzia dei Servizi Sanitari Regionali "Promozione della salute e gestione del rischio", si manifesta la necessità di un maggior coordinamento delle attività di gestione del rischio clinico a livello regionale e nazionale.

Sono ancora poche le Regioni che possono contare su un sistema regionale di gestione del rischio, mentre la sensibilità e l'attenzione dei professionisti sul tema della sicurezza del paziente è oggi elevata, non solo per il timore sempre maggiore delle conseguenze giudiziarie, ma anche perché si avverte un bisogno di cambiamento rispetto a una nuova etica medica e senso di responsabilità professionale. Se vogliamo raggiungere il traguardo di un servizio sanitario più affidabile e sicuro, dobbiamo essere consapevoli che «i problemi di oggi non saranno mai risolti nell'ambito della stessa cultura che li ha generati» (Albert Einstein).

\section{BIBLIOGRAFIA}

1. Baglioni A, Tartaglia R. Ergonomia e ospedale. Milano: Edizioni Il Sole 24 Ore, 2002

2. Tartaglia R, Bagnara S, Bellandi T, Albolino S. Healthcare ergonomics and patient safety. London: Taylor\&Francis, 2005

3. Nuti S, Tartaglia R, Niccolai F. Rischio clinico e sicurezza del paziente. Modelli e soluzioni. Bologna: Il Mulino, 2007 